\title{
Estudio de susceptibilidad antimicrobiana de Salmonella enterica en muestras de origen animal y alimentario
}

\author{
TANIA JUNOD ${ }^{a}$, JUANA LÓPEZ-MARTIN ${ }^{a}$, PAULA GÄDICKE ${ }^{a}$
}

Departamento Patología

y Medicina Preventiva, Universidad de Concepción,

Campus Chillán.

${ }^{a}$ Médico veterinario.

Recibido el 25 de abril de

2012, aceptado el $15 \mathrm{de}$ noviembre de 2012

Correspondencia a: Tania Junod

Av. Vicente Méndez 595, Chillán, Chile.

Fono: $56-42-208908$

Fax: 56-42-273201,

E-mail: taniajunod@udec.cl
$\mathrm{E}$ 1 género Salmonella presenta más de 2.500 serovares diferentes, algunos con gran virulencia y resistencia a múltiples antibióticos ${ }^{1,2,3}$. La amplia distribución de Salmonella enterica en el entorno, su prevalencia en la cadena alimentaria global, su virulencia y adaptabilidad tienen un enorme impacto en medicina, en salud pública y en la economía, dado el incremento de los brotes que se han presentado tanto en Chile como a nivel mundial ${ }^{4,5}$. El principal reservorio de Salmonella corresponde al tracto gastrointestinal de mamíferos, reptiles, aves e insectos, domesticados y salvajes ${ }^{6,7,8}$, estos animales jugarían un importante rol en la diseminación de este microorganismo en el medioambiente ${ }^{9}$. El uso indiscriminado de antibióticos en animales puede aumentar la selección de bacterias resistentes, que no sólo pueden infectar al hombre sino también causarle enfermedad. Se ha evidenciado la asociación entre el uso de antibióticos en alimentos animales con la resistencia de Salmonella aisladas en humanos ${ }^{10}$. Esto trae consecuencias sobre la salud humana como infecciones de mayor severidad y aumento de la frecuencia en tratamientos fallidos ${ }^{10}$. Muchos microorganismos presentan resistencia a múltiples antibióticos, y debido a que la diseminación de genes de resistencia puede ocurrir entre cepas bacterianas de distinta especie y origen, el impacto en salud pública es relevante ${ }^{11,12}$.

Existen programas de vigilancia de la resistencia antibiótica orientados principalmente a patógenos humanos, agentes causantes de zoonosis y 
bacterias indicadoras ${ }^{13}$. Como un aporte en esta área, el objetivo del presente trabajo fue determinar la susceptibilidad y patrones de resistencia a diferentes antibióticos en cepas de Salmonella enterica aisladas en muestras de origen alimentario y animal. Esto dado que la resistencia en estos microorganismos va en aumento y es de vital importancia el conocer la situación en nuestro país para tomar medidas cautelares e idear estrategias que minimicen la presentación de resistencia tanto en antibióticos de uso veterinario como de uso humano.

\section{Material y Métodos}

\section{Origen de las muestras}

Se analizaron todos los aislados bacterianos obtenidos de muestras ingresadas al laboratorio de Microbiología Clínica de la Facultad de Ciencias Veterinaria de la Universidad de Concepción, durante dos años (junio de 2007 a junio de 2009).

\section{Aislamiento y tipificación de Salmonella spp.}

Se utilizó el método bacteriológico tradicional $(\text { FDA-BAM })^{14}$. Las muestras fueron sometidas a un preenriquecimiento en Agua Peptona tamponada $\left(\right.$ Merck $\left.^{\circledR}\right)$ por $24 \mathrm{~h} \mathrm{a} 37^{\circ} \mathrm{C}$, luego un enriquecimiento selectivo en caldo Tetrationato/ caldo Rappaport por $24 \mathrm{~h}$ a $37^{\circ} \mathrm{C}$, finalmente las muestras fueron traspasadas a placas de Agar XLD con Novobiocina $(15 \mu \mathrm{m} / \mathrm{ml})$ (Merck $\left.{ }^{\circledR}\right)$ por $24-48 \mathrm{~h}$ a $42^{\circ} \mathrm{C}$. Las colonias sospechosas de Salmonella se sometieron a una confirmación fenotípica en TSI, LIA, Citrato de Simmons y SIM (Merck ${ }^{\circledR}$ ) a $37^{\circ} \mathrm{C}$ por $24-48 \mathrm{~h}$ y a confirmación serológica por aglutinación con suero polivalente O. Las cepas positivas fueron enviadas al Instituto de Salud Pública de Chile (ISP) para su serotipificación. Además, una vez aisladas se mantuvieron en cepario a $-20^{\circ} \mathrm{C}$.

\section{Pruebas para determinar susceptibilidad antibiótica}

Una vez tipificadas, las cepas aisladas durante el período fueron testeadas mediante método KirbyBauer bajo normas del Clinical and Laboratory Standards Institute (CLSI, 2008), para verificar susceptibilidad a los siguientes antibióticos: enrofloxacino $30 \mu \mathrm{g}$, oxitetraciclina $30 \mu \mathrm{g}$, ceftiofur $30 \mu \mathrm{g}$, ampicilina $10 \mu \mathrm{g}$, sulfametoxazol-trimetropim $25 \mu \mathrm{g}$, amoxicilina $20 \mu \mathrm{g}$, y florfenicol $30 \mu \mathrm{g}$.
Los puntos de corte de resistencia se basaron en los especificados por CLSI (2008) (Tabla 1). Como control de calidad, se utilizó la cepa E. coli ATCC $25922^{15}$. Las cepas que presentaron resistencia a tres o más antibióticos de grupos farmacológicos no relacionados se consideraron como resistentes a múltiples drogas (MDR), con estos antecedentes se establecieron patrones según serovares.

A todas aquellas cepas de Salmonella enterica que resultaron resistentes mediante el método Kirby-Bauer frente a uno o más de los antibióticos analizados, se les realizó concentración mínima inhibitoria (CMI) mediante método de macro dilución en series de caldo Mueller Hinton (Merck ${ }^{\circledR}$ ), de acuerdo a las indicaciones del CLSI(2008). Se preparó una solución madre de cada uno de los antibióticos y la lectura de los tubos fue realizada luego de su siembra e incubación por $24 \mathrm{~h}$ a $37^{\circ} \mathrm{C}$ en caldo Mueller-Hinton expresada en $\mu \mathrm{g} / \mathrm{ml}^{15}$.

\section{Análisis estadístico}

Para relacionar resistencia de cada antibiótico con el serovar de Salmonella y el origen de aislamiento, se realizaron regresiones logísticas multivariadas para el total de las cepas aisladas utilizando el software Stata SE 10.0 para Windows ${ }^{16}$, donde la variable dependiente fue la resistencia a cada antibiótico y las variables independientes el origen y serovar de las cepas; estas variables se probaron como confusores y se buscaron interacciones entre ellas, luego se construyeron los modelos en forma retrograda ${ }^{17}$. Las diferencias entre las medianas de CMI de cepas resistentes (según método Kirby-Bauer) entre los serovares y orígenes de aislamiento, se establecieron mediante test de Kruskall-Wallis, cuando existió un número adecuado de datos. Se realizó el cálculo de la $\mathrm{CIM}_{50} \mathrm{y} \mathrm{CIM}_{90}$. Además, para buscar los grupos de resistencia a antibióticos más relacionados con los serovares y orígenes se realizó un análisis de correspondencias múltiples (MCA), en el cual se grafican las dos dimensiones que agrupan la mayor variabilidad de los datos ${ }^{18}$, utilizando SE 10.0 para Windows.

\section{Resultados}

\section{Aislamiento y tipificación de cepas}

Se obtuvieron un total de 68 aislados de Salmonella enterica provenientes de bovinos, equinos, cerdos, gaviotas y alimentos, en el laboratorio de 
Tabla 1. Antibióticos y concentraciones utilizadas para las pruebas de susceptibilidad en Salmonella entérica

\begin{tabular}{|c|c|c|c|c|c|}
\hline \multirow{3}{*}{ Antibiótico } & \multirow{3}{*}{ Abreviación } & \multicolumn{4}{|c|}{ Niveles de susceptibilidad y resistenciaa } \\
\hline & & \multicolumn{2}{|c|}{ Kirby Bauer } & \multicolumn{2}{|c|}{ CMI } \\
\hline & & $\begin{array}{l}\text { Resistente a } \\
\quad(\mathbf{m m})\end{array}$ & $\begin{array}{l}\text { Sensible a } \\
(\mathrm{mm})\end{array}$ & $\begin{array}{c}\text { Resistente a } \\
(\mu \mathrm{m} / \mathrm{ml})\end{array}$ & $\begin{array}{c}\text { Sensible a } \\
(\mu \mathrm{m} / \mathrm{ml})\end{array}$ \\
\hline Enrofloxacino & Enr & $\leq 16^{b}$ & $\geq 23^{b}$ & $\geq 4^{b}$ & $\leq 1^{b}$ \\
\hline Oxitetraciclina & Oxi & $\leq 14$ & $\geq 19$ & $\geq 16$ & $\leq 4$ \\
\hline Ceftiofur & Cef & $\leq 17^{b}$ & $\geq 21^{b}$ & $\geq 32^{b}$ & $\leq 8^{\mathrm{b}}$ \\
\hline Ampicilina & Amp & $\leq 13$ & $\geq 17$ & NT & NT \\
\hline Sulfametoxazol-trimetoprim & SuTri & $\leq 10$ & $\geq 16$ & NT & NT \\
\hline Sulfadiazina & Sul & NT & NT & $\geq 512$ & $\leq 256$ \\
\hline Trimetoprim & Tri & NT & NT & $\geq 16$ & $\leq 8$ \\
\hline Amoxicilina & Amx & $\leq 13$ & $\geq 17$ & $\geq 32 / 16$ & $\leq 8 / 4$ \\
\hline Florfenicol & Flo & $\leq 12^{b}$ & $\geq 18^{b}$ & $\geq 32^{b}$ & $\leq 8^{b}$ \\
\hline Flumequina & Flu & NT & NT & $\geq 4^{b}$ & $\leq 1^{b}$ \\
\hline
\end{tabular}

NT: no testeado mediante este método. a: Los niveles de susceptibilidad y resistencia son los especificados en el CLSI (CLSI, 2008). b: No hay criterio de interpretación en el CLSI, las cepas se consideran resistentes a las concentraciones indicadas.

Tabla 2. Serovares positivos de S. entérica según origen de la muestra

\begin{tabular}{|c|c|c|c|c|c|c|}
\hline \multirow[t]{2}{*}{ Cepa bacteriana } & \multicolumn{6}{|c|}{ n positivos } \\
\hline & Alimento & Bovino & Equino & Cerdo & Gaviota & Total \\
\hline S. Thyphimurium & 0 & 3 & 2 & 4 & 0 & 9 \\
\hline S. Worthington & 0 & 0 & 0 & 1 & 0 & 1 \\
\hline S. Infantis & 1 & 0 & 0 & 23 & 0 & 24 \\
\hline S. GRUPO E (3,9:-:-) & 0 & 0 & 0 & 16 & 0 & 16 \\
\hline S. Enteritidis & 0 & 0 & 0 & 0 & 10 & 10 \\
\hline S. Anatum & 0 & 0 & 0 & 1 & 0 & 1 \\
\hline S. Derby & 2 & 0 & 0 & 2 & 0 & 4 \\
\hline S. Senftenberg & 2 & 0 & 0 & 0 & 0 & 2 \\
\hline S. Cholerasuis & 0 & 0 & 0 & 1 & 0 & 1 \\
\hline Total por cepa & 5 & 3 & 2 & 48 & 10 & 68 \\
\hline
\end{tabular}

Microbiología Clínica de la Facultad de Ciencias Veterinaria de la Universidad de Concepción. Se identificaron 9 serovares de Salmonella (Tabla 2). El 92,65\% provenía de aislados de origen animal y el resto de origen alimentario. De los 63 aislados de origen animal 76,19\% provenía de cerdos, $15,87 \%$ de gaviotas, $4,76 \%$ de bovinos y $3,17 \%$ de equinos.

Los aislados de Salmonella thyphymurium de equino y porcino provenían de tracto gastrointestinal, y las de bovino provenían de bazo e hígado. Para S. infantis aislado en alimento, correspondía a un alimento destinado a consumo animal. Todas las Salmonella enteritidis provenía de tracto gastrointestinal. Uno de Salmonella Derby fue aislada de un alimento destinado a consumo humano, mientras que la otra fue de alimento destinado a consumo animal. Las dos cepas de S. Senftenberg 
Tabla 3. Patrones MDR en serovares de Salmonella (Método Kirby Bauer)

\begin{tabular}{|c|c|c|c|}
\hline \multirow[t]{2}{*}{ Serovar } & \multicolumn{2}{|c|}{ n aislados } & \multirow{2}{*}{$\begin{array}{l}\text { Patrones de resistencia antimicrobiana } \\
\text { MDR (número de serovares resistentes) }\end{array}$} \\
\hline & Analizados & $\begin{array}{l}\text { Resistentes (uno o } \\
\text { más antibióticos) }\end{array}$ & \\
\hline S. Thyphimurium & 9 & 6 & $\begin{array}{l}\text { SuTri, Oxi, Amx, Amp (1) } \\
\text { SuTri, Oxi, Cef (1) }\end{array}$ \\
\hline S. GRUPO E $(3,9 ;-;-)$ & 16 & 14 & $\begin{array}{l}\text { Oxi, Amx, Cef, Enr, Amp (1) } \\
\text { SuTri, Oxi, Amx, Enr, Amp (1) } \\
\text { SuTri, Oxi, Flo, Amx, Amp (2) } \\
\text { SuTri, Oxi, Cef (1) } \\
\text { SuTri, Oxi, Amx, Cef, Amp (1) } \\
\text { SuTri, Oxi, Flo (1) }\end{array}$ \\
\hline S. Derby & 4 & 4 & $\begin{array}{l}\text { SuTri, Oxi, Flo, Amx, Cef, Enr, Amp (1) } \\
\text { SuTri, Oxi, Cef (1) }\end{array}$ \\
\hline
\end{tabular}

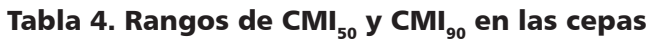
de Salmonella $(n=68)$

\begin{tabular}{|c|c|c|}
\hline Antibiótico & $\begin{array}{c}\mathrm{CMI}_{50} \\
(\mu \mathrm{g} / \mathrm{mL})\end{array}$ & $\begin{array}{c}\mathrm{CMI}_{90} \\
(\mu \mathrm{g} / \mathrm{mL})\end{array}$ \\
\hline Enrofloxacino & 2 & 16 \\
\hline Florfenicol & 32 & 128 \\
\hline Oxitetraciclina & 256 & 512 \\
\hline Sulfadoxina & 256 & 512 \\
\hline Flumequina & 128 & 512 \\
\hline Trimetoprim & 1.024 & 1.024 \\
\hline Amoxicilina & 512 & 1.024 \\
\hline Ceftiofur & 16 & 16 \\
\hline
\end{tabular}

fueron aisladas desde alimento destinado a consumo humano.

\section{Susceptibilidad antibiótica}

En las pruebas de susceptibilidad antibiótica (método Kirby Bauer), 34 cepas (50\%) fueron resistentes a alguno de los antibióticos utilizados en el estudio, los cuales son de uso en terapia veterinaria y humana (Tabla 3). El 69,1\% de las cepas presentó resistencia a oxitetraciclina.

Los aislados de S. enteritidis, S. Worthingthon y $S$. anatum fueron susceptibles a todos los antibióticos analizados en este estudio.

El 16,18\% de las cepas analizadas presentaron MDR, que correspondió a 3 de los serovares aislados en este estudio: S. thyphimurium (22,2\%),
S. Grupo E (3,6,:-:-) $(43,75 \%)$ y S. Derby $(50 \%)$ (Tabla 3), siendo el $90,91 \%$ proveniente de porcinos. Se encontraron 11 patrones diferentes que incluyeron un mínimo de tres antibióticos y máximo de siete. El patrón más frecuente incluyó tres antibióticos: Cef,Oxi,STX; encontradas en $S$. Derby, S. thyphimurium y S. Grupo E (3,9:-:-).

El modelo de regresión logística, que presentó relación significativa, indica que S. Grupo E (3,9::-), tuvo más riesgo de ser resistente a oxitetraciclina que $S$. thyphimurium (OR: 7,7; IC95\% 1,15; $51,17)(\mathrm{p}<0,05)$ para este grupo de cepas, mientras que los orígenes no demostraron asociación significativa con el riesgo de resistencia $(\mathrm{p}>0,05)$.

El análisis de CMI para oxitetraciclina (30 cepas) indicó $96,66 \%$ de serovares resistentes. Los serovares S. Derby y $S$. thyphimurium, ambas aisladas de cerdos, fueron resistentes a ceftiofur. Enrofloxacino presentó 33,33\% de los serovares resistentes, también provenientes de cerdos. Para el caso de amoxicilina, todos los serovares fueron resistentes según el análisis de CMI, mientras que para florfenicol se observó $60 \%$ de serovares resistentes. Para sulfadiazina (12 cepas) hubo $25 \%$ de serovares resistentes, mientras que flumequina (68 cepas) presentó 95,58\% de serovares resistentes. Los rangos de $\mathrm{CMI}_{50}$ y $\mathrm{CMI}_{90}$ se observan en la Tabla 4.

El análisis gráfico exploratorio (MCA) que en sus dos dimensiones relaciona la resistencia y origen de las cepas, explican $91,7 \%$ de la variabilidad total (Figura 1). Se observó que las cepas resistentes se agrupan hacia la izquierda, en relación a las cepas susceptibles que se agrupan en 


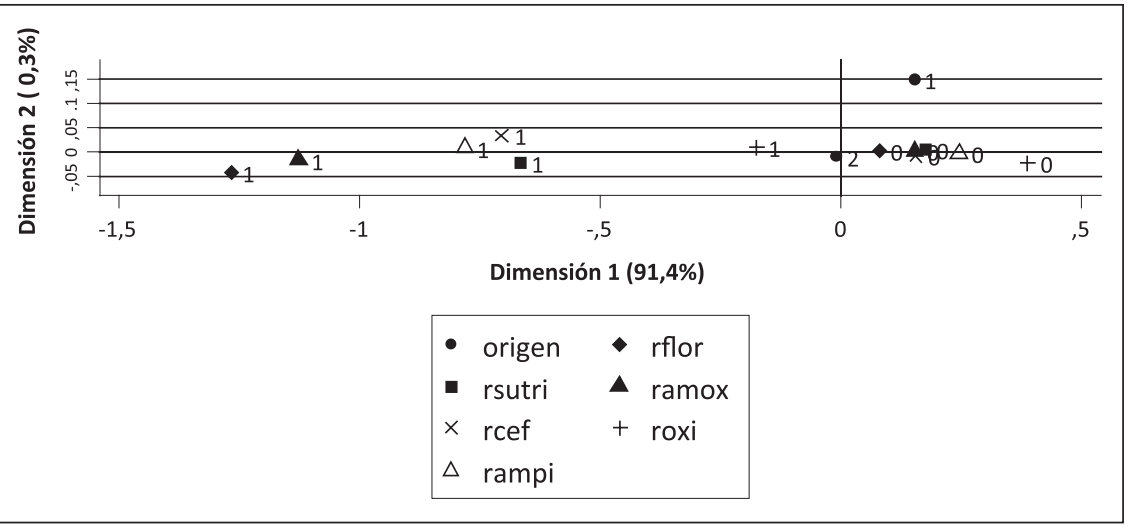

Figura 1. Relación entre susceptibilidad antibiótica y origen de las cepas de Salmonella. 0: susceptible; 1: resistente para cada uno de los antibióticos. Origen: 1: Alimentario; 2: Animal. el eje hacia la derecha, explicado en $91,4 \%$ por la dimensión 1. El origen alimentario difiere del origen animal, al encontrarse alejado del eje y de los otros puntos, su variabilidad está dada por la dimensión 2. Esto indicaría que las resistencias y susceptibilidades pudiesen asociarse a algún tipo de factor o efecto, dado que todas estas variables no estarían actuando de forma independiente.

\section{Discusión}

Los diferentes serovares aislados en este estudio corresponden a serovares no tifoideos de S. enteri$c a$, que son considerados zoonóticos en los países en desarrollo ${ }^{19}$. Los resultados de serotipificación muestran la existencia de una amplia variedad de

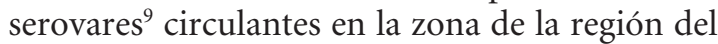
Bío Bío, tanto en animales y alimentos. En alimento se determinaron dos serovares, $S$. Derby que se describe habitualmente asociado a porcinos ${ }^{20}$ y $S$. Senfteberg. S. Derby en este estudio provenía de alimento destinado a aves en una crianza traspatio $^{21}$ en la que se encontraban otros animales de granja cercanos, por lo que se pudiese sospechar de una transmisión entre especies. El serovar $S$. Derby ha sido uno de los aislados más frecuentes en humanos ${ }^{22}$. Es importante entonces contar con alimentos inocuos para planteles animales y así asegurar el estatus sanitario de ellos y minimizar el riesgo de llegar al ser humano.

En el informe de la Red de Vigilancia de la Resistencia a los Antibióticos OPS del año 2004, en nuestro país los serovares más frecuentes en aislamientos de alimentos fueron: S.4,12:d:-, S. thyphimurium, S. enteritidis, S. Derby y S. Senf- tenberg. Este último, en el presente reporte, fue identificado como cepa MDR pese a que en el año 2004, de acuerdo a la OPS, no presentó resistencia frente a ninguno de los antibióticos utilizados. La resistencia de los aislados animales totales entregado por la OPS señala que los serovares más frecuentes fueron S.4,12:d:-, S. thyphimurium, S. enteritidis y $S$. infantis. Sólo algunas cepas de S.4,12:d:- presentaron resistencia a ampicilina, cloranfenicol, amoxicilina-ácido clavulánico y ácido nalidíxico ${ }^{23}$, que difiere de las resistencias encontradas en este reporte donde algunas cepas fueron MDR frente a antibióticos de las mismas familias, lo que apoya el hecho de un aumento de la resistencia en los aislados de Salmonella spp. Este comportamiento puede asociarse a la variabilidad en las poblaciones de Salmonella dado por patrones genéticos diversos. Esto se explica por la adquisición o presentación de diferentes mecanismos de resistencia dado por la diversidad de la población de Salmonella spp. presente.

En los serovares de origen animal predominan S. infantis y S. Grupo E (3,9;-;-) aisladas desde cerdos, lo cual indica que estos serovares en las distintas etapas de producción no son capaces de establecerse de forma endémica a diferencia de S. thyphimurium, S. cholerasuis o S. Derby entre otros $^{24}$. Hay que considerar también que algunos de los serovares analizados son de origen silvestre, aislados a partir de gaviotas, las cuales pueden actuar como diseminadoras de estos agentes hacia otros animales, incluido el hombre, o bien hacia alimentos.

Es importante destacar que una de las cepas aisladas corresponde al serovar Worthington, este serovar ha sido descrito en algunos países como 
Susceptibilidad antimicrobiana de Salmonella enterica - T. Junod et al

un patógeno emergente causal de meningitis neonatal ${ }^{25,26}$. Este serovar fue aislado de cerdo, con baja resistencia a antibióticos, por lo que la terapia antimicrobiana frente a éste en humanos, debiera dar buenos resultados.

Los resultados obtenidos de alta resistencia a oxitetraciclina $(69,1 \%)$ son similares a lo detectado por Alaniz ${ }^{27}$ que alcanzaba 57,4\% en Salmonella de origen animal. También es importante destacar que se encontraron resistencias a ampicilina, flumequina, ceftiofur y sulfadiazina-trimetoprim. Algunos de estos compuestos, junto a las tetraciclinas, han sido utilizados por décadas en la producción animal, ya sea como profilaxis o con fines terapéuticos, y en tratamiento de infecciones humanas por lo que su resistencia está ampliamente difundida ${ }^{28}$. Es por esto que estos tratamientos en la actualidad son poco efectivos, como por ejemplo el uso de amoxicilina en personas ${ }^{29}$.

Según el análisis exploratorio observado en la construcción del MCA, la variabilidad de el origen alimentario y animal indica que la mantención y diseminación de cepas resistentes se explicaría por factores como sistemas de producción, manejos de tratamientos antibióticos, contacto con reservorios naturales, entre otros ${ }^{30,31}$.

Los valores de CMI que superan los niveles mínimos de interpretación de resistencia antibiótica, se relacionan con la expresión de ciertas enzimas que deben ser detectadas mediante estudios moleculares ${ }^{32}$.

Dado que este es uno de los pocos estudios de caracterización de Salmonella enterica en el país, da una visión de la susceptibilidad antimicrobiana presentada por los aislados de origen animal y alimentario de forma transversal, pero como es un muestreo a conveniencia, es necesario realizar estudios que sean representativos a nivel poblacional para identificar los sectores más afectados e ir evaluando la evolución de la resistencia presentada principalmente en animales y ambiente. Además es importante contar con pruebas basadas en técnicas moleculares a fin de aportar mayor información para la caracterización y genotipificación de los aislados de Salmonella spp. en nuestro país.

\section{Conclusiones}

La tipificación de los aislados analizados en el estudio logró determinar 9 diferentes serovares potencialmente zoonóticos, los cuales presentan resistencia a uno o más de los antibióticos utilizados en el estudio, encontrándose además que $20,5 \%$ de las cepas presentan MDR, principalmente de cerdos y siendo oxitetraciclina la droga con mayor aislados resistentes $(69,1 \%)$.

El serovar $S$. infantis tiene una menor probabilidad de presentar resistencia a sulfa-trimetoprim que $S$. thyphimurium y el serovar S. grupo $\mathrm{E}$ $(3,9 ;-;-)$ tiene mayor riesgo de resistencia frente a ampicilina que $S$. thyphimurium para el grupo de cepas analizadas.

Agradecimientos: INNOVA BIOBIO y Laboratorio Veterquímica ${ }^{\circledR}$ por su disposición y aporte al desarrollo de esta investigación.

\section{Referencias}

1. Lauderdale TL, Frank M, Aarestrup, Pei-Chen Chen, Jui-Fen Lai, Hui-Ying Wang, et al. Multidrug resistance among different serotypes of clinical Salmonella isolates in Taiwan. Diagnostic Microbiology and Infectious Disease 2006; 55: 149-55.

2. Kim HJ, Si-Hong P, Hae-Yeong K. Comparison of Salmonella enterica Serovar Typhimurium LT2 and NonLT2 Salmonella Genomic Sequences, and Genotyping of Salmonellae by Using PCR. Applied and Environmental Microbiology 2006; 72 (9): 6142-51.

3. Zhao S, McDermott PF, White DG, Qaiyumi S, Friedman SL, Abbott JW, et al. Characterization of multidrug resistant Salmonella recovered from diseased animals. Vet Microbiol 2007; 123: 122-32.

4. Whiting RC, Hogue A, Schlosser WD, Ebel ED, Morales RA, Baker A, et al. A quantitative process model for Salmonella enteritidis in shell eggs. J Food Sci 2000; 65 (5): 864-9.

5. Fica AM, Alexandres S, Prat A, Fernández J, Fernández I, Heitmann. Cambios epidemiológicos de las salmonelosis en Chile. Desde Salmonella typhi a Salmonella enteritidis. Rev Chil Infect 2001; 18 (2): 85-93.

6. Toro HC, Saucedo C, Borie RE, Gough H, Alcaíno. Status of free-living Pigeons in the city of Santiago. Avian. Pathol 1999; 28: 619-23.

7. Wray RM, Davies DM. Control ambiental de Salmonella. Avicultura Profesional 2000; 18 (5): 18-21.

8. González-Acuña D, Silva F, Moreno L, Cerda F, Donoso S, Cabello J, et al. Detección de algunos agentes zoonóticos en la paloma doméstica (Columba livia) en la ciudad de Chillán, Chile. Rev Chil Infect 2007; 24 (3): 199-203. 
9. Gopee NV, Adesiyun AA, Caesar K. Retrospective and longitudinal study of salmonellosis in captive wildlife in Trinidad. J Wildl Dis 2000; 36: 284-93.

10. Angulo FJ, Nargund VN, Chiller TC. Evidence of an association between use of anti-microbial agents in food animals and anti-microbial resistance among bacteria isolated from humans and the human health consequences of such resistance. J Vet Med B Infect Dis Vet Public Health 2004a; 51 (8-9): 374-9.

11. Rice LB. Bacterial Monopolist: The Bundling an Disemination of antimicrobial Resistance Genes in Gram Positive Bacteria. Clinical Infectious Diseases 2000; 31: 762-9.

12. Van den Bogaard AE, Willems R, London N, Top J, Stobberingh EE. Antibiotic resistance of faecal enterococci in poultry, farmers and poultry slaughterers. J Of Antimicrob Chemother 2002; 49: 497-505.

13. Lanz R, Kuhnert, Boerlin P. Antimicrobial resistance and resistance gene determinant in clinical Escherichia coli from different animal species in Switzerland. Vet Microbiol 2002; 91: 73-84.

14. FDA (Food and Drug Administration). Bacteriologycal Analytical Manual (BAM), ${ }^{\text {th }}$ Edition. 1995. Chapter 5. Salmonella. En: Food Borne Pathogens Monograph Number 1 Salmonella. 1997. Oxoid.

15. CLSI (Clinical and Laboratory Standards Institute). Performance standards for antimicrobial disk and dilution susceptibility tests for bacteria isolated from animals; approved standard- third edition 2008; 28 (8).

16. StataCorp. Stata Statistical Software/SE 10.0 for Windows: College Station, TX: 543 StataCorp. LP., 2007.

17. Dohoo IW, Martin, Stryhn H. Veterinary Epidemiologic Research. National Library of Canada Cataloghuing in Publication. 2003.

18. Vivanco M. Análisis estadístico multivariable. Teoría y Práctica. Editorial Universitaria, Santiago de Chile. 1999.

19. Threlfalla EJ. Antimicrobial Drug resistance in Salmonella: problems and perspectives in food-and water-borne infection. FEMS Microbiol Rev 2002; 26: 141-8.

20. Ibar MP, Vigo G, Piñeyro P, Caffer MI, Quiroga P, Perfumo C, et al. Serovariedades de Salmonella enterica subespecie enterica en porcinos de faena y su resistencia a los antimicrobianos. Revista Argentina de Microbiología 2009; 41: 156-62.

21. Troncoso L. Detección de Salmonella spp. en crianzas de gallina de tipo artesanal de la provincia de Nuble (Chile). Memoria de Título, Facultad Ciencias Veterinarias, Universidad de Concepción. 2007.

22. Usera MA, Aladueña A, Días R, De La Fuente M, Cerdán P, Gutiérrez R, et al. Análisis de las cepas de Salmonella spp. aisladas de muestras de origen humano en España en el año 2001. Boletín Epidemiológico Semanal (España) $2003 ; 11: 133-44$

23. OPS/HDM/CD/A/408/06. 2005. Informe Annual de la Red de Monitoreo/Vigilancia de la Resistencia a los Antibióticos-2004-Brasilia-Brazil 27 al 29 de julio.

24. Baggesen DL, Wegener HC, Bager F, Stege H, Christensen J. Herd Prevalence of Salmonella enterica infections in Danish slaughter pigs determined by microbiological testing. Preventive Veterinary Medicine 1996; 26: 201 13.

25. Ghadage DP, Bal AM. Antibiotic Susceptibility Pattern of Salmonella worthingthon Isolated from Neonates-A Retrospective Study. Jpn J Infect Dis 2002; 55: 45-6.

26. Muley VA, Pol SS, Dohe VB, Nagdawane RP, Arjunwadkar VP, Pandit DP, et al. Neonatal outbreak of Salmonella worthington in a general hospital. Indian J Med. Microbiol 2004; 22 (1): 51-3.

27. Alaniz RO, Ibarra MLR, Barbosa BTR, Morales ALJ. Resistencia a antimicrobianos de cepas de Salmonella aisladas de fuentes animales. Vet Méx 1997; 28: 215-20.

28. Breuil J, Brisabois A, Casin I, Armand-Lefevre L, Frémy S, Collatz E. Antibiotic resistance in Salmonellae isolates from humans and animals in France: comparative data from 1994 and 1997. Journal of Antimicrobial Chemotherapy 2000; 46: 965-71.

29. Gebreyes WA, Altiera C. Molecular Characterization of Multidrug-Resistant Salmonella enterica subsp. enterica Serovar Typhimurium Isolates from Swine. Journal Of Clinical Microbiolog 2002; 40 (8): 2813-22.

30. Nollet N, Houf K, Dewulf J, De Kruif A, De Zutter L, Maes D. Salmonella in sows: a longitudinal study in farrow-to-finish pig herds. Vet Res 2005; 36: 645-56.

31. García-Feliz C, Carvajal A, Collazos JA, Rubio P. Herdlevel risk factors for faecal shedding of Salmonella enterica in Spanish fattening pigs. Preventive Veterinary Medicine 2009; 91: 130-6.

32. Tenover FC, Mohamed MJ, Gorton TS, Dembek ZF. Detection and Reporting of Organism Producing Extended-Spectrum $\beta$-Lactamases: Survey of Laboratories in Connecticut. J Clin Microbiol 1999; 37: 4065-70. 